\title{
The empirical process of a short-range dependent stationary sequence under Gaussian subordination
}

\author{
Sándor Csörgö1,^, Jan Mielniczuk ${ }^{2}$ \\ 1 Department of Statistics, The University of Michigan, 1440 Mason Hall, Ann Arbor, \\ MI 48109-1027, USA (e-mail: scsorgo@stat.lsa.umich.edu) \\ 2 Institute of Computer Science, Polish Academy of Sciences, Ordona 21, PL-01-237 Warsaw, \\ Poland (e-mail: miel@IPIPAN.Waw.PL)
}

Received: 1 April 1994 / In revised form: 1 June 1995

Summary. Consider the stationary sequence $X_{1}=G\left(Z_{1}\right), X_{2}=G\left(Z_{2}\right), \ldots$, where $G(\cdot)$ is an arbitrary Borel function and $Z_{1}, Z_{2}, \ldots$ is a mean-zero stationary Gaussian sequence with covariance function $r(k)=E\left(Z_{1} Z_{k+1}\right)$ satisfying $r(0)=1$ and $\Sigma_{k=1}^{\infty}|r(k)|^{m}<\infty$, where, with $I\{\cdot\}$ denoting the indicator function and $F(\cdot)$ the continuous marginal distribution function of the sequence $\left\{X_{n}\right\}$, the integer $m$ is the Hermite rank of the family $\{I\{G(\cdot) \leqq x\}-$ $F(x): x \in \mathbb{R}\}$. Let $F_{n}(\cdot)$ be the empirical distribution function of $X_{1}, \ldots, X_{n}$. We prove that, as $n \rightarrow \infty$, the empirical process $n^{1 / 2}\left\{F_{n}(\cdot)-F(\cdot)\right\}$ converges in distribution to a Gaussian process in the space $\mathscr{D}[-\infty, \infty]$.

Mathematics Subject Classification (1991): 60F17, 62G30

\section{Introduction}

Let $Z$ be a standard normal random variable. If $H: \mathbb{R} \mapsto \mathbb{R}$ is a Borel measurable function such that $E(H(Z))=0$ and $E\left(H^{2}(Z)\right)<\infty$, and $H_{k}(y):=$ $(-1)^{k} e^{y^{2} / 2} d^{k} e^{-y^{2} / 2} / d y^{k}, y \in \mathbb{R}, k=0,1,2, \ldots$, denotes the $k$ th Hermite polynomial, then $H(\cdot)$ admits the Fourier-Hermite expansion $H(y)=$ $\Sigma_{k=m(H)}^{\infty} J_{k} H_{k}(y) / k !, y \in \mathbb{R}$, in the weighted $\mathscr{L}^{2}$ space $\mathscr{L}^{2}(\mathbb{R}, \varphi)$, where $\varphi(\cdot)$ is the standard normal density, $J_{k}:=E\left(H_{k}(Z) H(Z)\right)$, and the integer $m(H) \in \mathbb{N}$, the Hermite rank of $H(\cdot)$, is the index of the first non-zero coefficient $J_{k}, k \in \mathbb{N}$. (Since we assume that $E(H(Z))=0$, we have $J_{0}=0$.)

Let, throughout this paper, $Z_{1}, Z_{2}, \ldots$ be a stationary Gaussian sequence with

$$
E\left(Z_{1}\right)=0, \quad E\left(Z_{1}^{2}\right)=1 \quad \text { and } \quad r(k):=E\left(Z_{1} Z_{k+1}\right), \quad k \in \mathbb{N} .
$$

* Partially supported by NSF Grant DMS-9208067 
Assuming that $H(\cdot)$ is as above and that the sequence $\{r(k)\}_{k=1}^{\infty}$ is regularly varying at infinity with index $-\alpha$, where $0<\alpha<1$, Dobrushin and Major [5] and Taqqu [11] have shown that under the long-range dependence condition $m(H) \alpha<1$, so that $\Sigma_{k=1}^{\infty}|r(k)|^{m(H)}=\infty$, the suitably normed partial-sum process based on the sequence $H\left(Z_{1}\right), H\left(Z_{2}\right), \ldots$ converges in distribution to the so-called Hermite process $Y_{m(H)}(\cdot)$ of rank $m(H)$. Here $Y_{1}(\cdot)$ is fractional Brownian motion, but the processes $Y_{2}(\cdot), Y_{3}(\cdot), \ldots$ are no longer Gaussian. On the other hand, not assuming the regular variation of $\{r(k)\}_{k=1}^{\infty}$ and citing earlier references, Breuer and Major [2] and Giraitis and Surgailis [7] have independently shown that under the natural short-range dependence condition that $\Sigma_{k=1}^{\infty}|r(k)|^{m(H)}<\infty$, the asymptotic behavior of the partial-sum process, based on $H\left(Z_{1}\right), H\left(Z_{2}\right), \ldots$, is qualitatively the same as in weakly dependent situations. Namely, the required norming is the traditional $\sqrt{n}$, and the finite-dimensional distributions of the process converge to those of a Gaussian process.

Consider now an arbitrary Borel measurable function $G: \mathbb{R} \mapsto \mathbb{R}$, and let

$$
X_{1}:=G\left(Z_{1}\right), \quad X_{2}:=G\left(Z_{2}\right), \ldots, \quad \text { and } \quad F(x):=P\{G(Z) \leqq x\}, \quad x \in \mathbb{R} .
$$

Let $I\{\cdot\}$ denote the indicator function and set $F_{n}(x):=n^{-1} \Sigma_{k=1}^{n} I\left\{X_{k} \leqq\right.$ $x\}$ for the $n$th empirical distribution function of the subordinated sequence $\left\{X_{k}\right\}_{k=1}^{\infty}$, so that

$$
F_{n}(x)-F(x)=\frac{1}{n} \sum_{k=1}^{n}\left[I\left\{X_{k} \leqq x\right\}-F(x)\right]=\frac{1}{n} \sum_{k=1}^{n} D_{x}\left(Z_{k}\right), \quad x \in \mathbb{R}, n \in \mathbb{N},
$$

where, with $m\left(D_{x}\right) \in \mathbb{N}$ as the Hermite rank of the function $D_{x}(\cdot)$, again in $\mathscr{L}^{2}(\mathbb{R}, \varphi)$,

$$
\begin{aligned}
D_{x}(\cdot) & :=G_{x}(\cdot)-F(x):=I\{G(\cdot) \leqq x\}-F(x) \\
& =\sum_{k=m\left(D_{x}\right)}^{\infty} \frac{J_{k}(x)}{k !} H_{k}(\cdot), \quad x \in \mathbb{R},
\end{aligned}
$$

where $J_{k}(x)=E\left(H_{k}(Z) D_{x}(Z)\right)=E\left(H_{k}(Z) G_{x}(Z)\right)$ for all $k \in \mathbb{N}$. Finally, the Hermite rank of the family $\left\{D_{x}(\cdot): x \in \mathbb{R}\right\}$ of functions is defined as

$$
m:=\min \left\{m\left(D_{x}\right): x \in S_{F}\right\} \in \mathbb{N}, \quad \text { where } S_{F}:=\{x \in \mathbb{R}: 0<F(x)<1\} .
$$

Complementing the results for partial sums under long-range dependence, Dehling and Taqqu [3] have proved that under the same condition of regular variation and $m \alpha<1$, so that $\sum_{k=1}^{\infty}|r(k)|^{m}=\infty$, the suitably normed empirical process $F_{n}(\cdot)-F(\cdot)$ converges in distribution in the space $\mathscr{D}[-\infty, \infty]$ to a degenerate process $J_{m}(\cdot) Y_{m}(1) / m !$. Such an asymptotic behavior is of course strikingly different from that in independent or weakly dependent situations. Here and in what follows, $\mathscr{D}[-\infty, \infty]$ denotes the non-separable metric space of all real functions defined on $[-\infty, \infty]$ that are right-continuous everywhere in $\{-\infty\} \cup \mathbb{R}$ and have left-side limits everywhere in $\mathbb{R} \cup\{\infty\}$ such that the distance of $g, h \in \mathscr{D}[-\infty, \infty]$ is $\sup \{|g(x)-h(x)|: x \in\{-\infty\} \cup \mathbb{R} \cup\{\infty\}\}$. Convergence in distribution, denoted by $\stackrel{\mathscr{D}}{\rightarrow}$, in this space is meant with respect 
to the $\sigma$-algebra generated by the set of open balls for this metric. The space $\mathscr{D}[0,1]$ and weak convergence in $\mathscr{D}[0,1]$ is defined analogously, and $\mathscr{C}[0,1]$ is the subspace of $\mathscr{D}[0,1]$ consisting of continuous functions. Unspecified convergence relations are meant as $n \rightarrow \infty$.

Our aim is to round off the study by proving that under the natural analogue of the Breuer-Major [2] and Giraitis-Surgailis [7] condition of short-range dependence, condition (2.1) below, the empirical process $\sqrt{n}\left[F_{n}(\cdot)-F(\cdot)\right]$ converges in distribution in $\mathscr{D}[-\infty, \infty]$ to a Gaussian process. The theorem in the next section complements the results for partial sums under short-range dependence in the Gaussian subordination model (1.1)-(1.5) exactly as the Dehling and Taqqu [3] convergence theorem complements those under longrange dependence. The proof is separated in a third section, following a discussion. We point out among others that the arising limiting process is the same as in the case of weak dependence defined by various mixing conditions on the sequence $\left\{X_{k}\right\}_{k=1}^{\infty}$, even though no form of mixing is assumed here, as obtained in Theorem 22.1 of Billingsley [1], Sen [9], Deo [4] and Gastwirth and Rubin [6].

\section{The result and discussion}

Consider the set-up and notation in (1.1) and (1.2)-(1.5). No condition of regular variation is required for the covariances $r(\cdot)$.

Theorem. Suppose that $F(\cdot)$ is continuous and the sequence $\{r(k)\}_{k=1}^{\infty}$ satisfies

$$
\sum_{k=1}^{\infty}|r(k)|^{m}<\infty
$$

Then $\sqrt{n}\left[F_{n}(\cdot)-F(\cdot)\right] \stackrel{\mathscr{D}}{\rightarrow} W(\cdot)$ in $\mathscr{D}[-\infty, \infty]$, where $W(\cdot)$ is a meanzero Gaussian process with covariance function

$$
E(W(x) W(y))=\sum_{q=m}^{\infty} \frac{J_{q}(x) J_{q}(y)}{q !}\left\{r(0)+2 \sum_{k=1}^{\infty} r^{q}(k)\right\}, \quad x, y \in \mathbb{R} .
$$

For $x \in \mathbb{R}$ and $k \in \mathbb{N}$, let $r_{x}(k)=E\left(D_{x}\left(Z_{1}\right) D_{x}\left(Z_{k+1}\right)\right)$, where $D_{x}(\cdot)$ is as in (1.4). Similarly as in the proof of Lemma 5 of Giraitis and Surgailis [7], it can be shown that if $r(n) \rightarrow 0$, then condition (2.1) is equivalent to the condition:

$$
\sum_{k=1}^{\infty}\left|r_{x}(k)\right|<\infty \text { for every } x \in \mathbb{R} .
$$

Indeed, by Mehler's classical formula for the Hermite expansion of a bivariate normal density,

$$
E\left(H_{l}\left(Z_{j}\right) H_{q}\left(Z_{k}\right)\right)=\delta_{l q} l ! r^{l}(j-k), \quad j, k, l, q \in \mathbb{N}, \quad k \leqq j,
$$

where $\delta_{l q}$ is Kronecker's delta, and by (1.4) this implies that

$$
r_{x}(k)=r^{m_{x}}(k) \sum_{q=m_{x}}^{\infty} \frac{J_{q}^{2}(x)}{q !} r^{q-m_{x}}(k), \quad x \in \mathbb{R}, k \in\{0,1,2, \ldots\},
$$


where $m_{x}:=m\left(D_{x}\right)$. Thus, setting $M_{k}(x):=\sum_{q=m_{x}+1}^{\infty} J_{q}^{2}(x) r^{q-m_{x}}(k) / q !$,

$$
|r(k)|^{m_{x}}\left[\frac{J_{m_{x}}^{2}(x)}{m_{x} !}-\left|M_{k}(x)\right|\right] \leqq\left|r_{x}(k)\right| \leqq|r(k)|^{m_{x}}\left[\frac{J_{m_{x}}^{2}(x)}{m_{x} !}+\left|M_{k}(x)\right|\right] .
$$

Since $\Sigma_{q=m_{x}}^{\infty} J_{q}^{2}(x) / q !=r_{x}(0)<\infty$ and $m_{x} \geqq m$, the condition that $r(n) \rightarrow 0$ yields

$$
\left|M_{k}(x)\right| \leqq \frac{1}{2} \frac{J_{m_{x}}^{2}(x)}{m_{x} !} \quad \text { and so } \quad|r(k)|^{m_{x}} \frac{1}{2} \frac{J_{m_{x}}^{2}(x)}{m_{x} !} \leqq\left|r_{x}(k)\right| \leqq|r(k)|^{m} \frac{3}{2} \frac{J_{m_{x}}^{2}(x)}{m_{x} !}
$$

for each $x \in \mathbb{R}$ if $k$ is sufficiently large. Substituting an $x_{0}$ in (1.5) for which $m_{x_{0}}=m\left(D_{x_{0}}\right)=m$, we see that (2.3) implies (2.1). The reverse implication is trivial.

So, condition (2.1) of the theorem, a necessary condition for $W(\cdot)$ to be well defined, can be stated without referring to the rank $m$ of the family $\left\{D_{x}(\cdot): x \in \mathbb{R}\right\}$. It is just the summability of the covariances of the terms in the representation of $n\left[F_{n}(\cdot)-F(\cdot)\right]$ from (1.3). However, the introduction of the Hermite rank is useful: it will play an important role in the proof. On the other hand, it is equally important to see that the value of $m$ does not enter in the form of the limiting process in contrast to the same estimation problem under long-range dependence, that is, as in the limiting process of Dehling and Taqqu [3] described above. It is also obvious from the equivalence of the conditions (2.1) and (2.3) that condition (2.1) is equivalent to the condition that $\sum_{k=1}^{\infty}\left|r_{x_{0}}(k)\right|<\infty$, where $x_{0}$ is such that $m\left(D_{x_{0}}\right)=m$, provided $r(n) \rightarrow 0$.

By (1.4) and (2.4), E( $\left.D_{x}\left(Z_{1}\right) D_{y}\left(Z_{1}\right)\right)=\min \{F(x), F(y)\}-F(x) F(y)$ and

$$
E\left(D_{x}\left(Z_{1}\right) D_{y}\left(Z_{k+1}\right)\right)=\sum_{q=m}^{\infty} \frac{J_{q}(x) J_{q}(y)}{q !} r^{q}(k), \quad x, y, \in \mathbb{R}, k \in\{0,1,2, \ldots\},
$$

extending (2.5). Hence, setting $d_{x}(\cdot):=I\{\cdot \leqq x\}-F(x)$, the covariance function (2.2) of the process $W(\cdot)$ can be written as

$$
\begin{aligned}
& E(W(x) W(y)) \\
& \quad=E\left(D_{x}\left(Z_{1}\right) D_{y}\left(Z_{1}\right)\right)+2 \sum_{k=1}^{\infty} E\left(D_{x}\left(Z_{1}\right) D_{y}\left(Z_{k+1}\right)\right) \\
& \quad=E\left(D_{x}\left(Z_{1}\right) D_{y}\left(Z_{1}\right)\right)+\sum_{k=1}^{\infty}\left[E\left(D_{x}\left(Z_{1}\right) D_{y}\left(Z_{k+1}\right)\right)+E\left(D_{y}\left(Z_{1}\right) D_{x}\left(Z_{k+1}\right)\right)\right] \\
& \quad=E\left(d_{x}\left(X_{1}\right) d_{y}\left(X_{1}\right)\right)+\sum_{k=1}^{\infty}\left[E\left(d_{x}\left(X_{1}\right) d_{y}\left(X_{k+1}\right)\right)+E\left(d_{y}\left(X_{1}\right) d_{x}\left(X_{k+1}\right)\right)\right]
\end{aligned}
$$

for all $x, y \in \mathbb{R}$. Thus the limiting process of the theorem coincides with the limiting process of the empirical process when certain mixing conditions are stipulated for the underlying stationary sequence, as in Theorem 22.1 of Billingsley [1], Sen [9] and Deo [4].

Let $\Phi(\cdot)$ be the standard normal distribution function. If $\left\{X_{k}\right\}_{k=1}^{\infty}$ is an arbitrary stationary sequence with a continuous and strictly increasing marginal 
distribution function $F(\cdot)$, then $X_{k}=G\left(Z_{k}^{*}\right)$, where $G(\cdot)=F^{-1}(\Phi(\cdot))$ and $Z_{k}^{*}=\Phi^{-1}\left(F\left(X_{k}\right)\right)$ is a standard normal random variable for each $k \in \mathbb{N}$. However, $\left\{Z_{k}^{*}\right\}_{k=1}^{\infty}$ is not necessarily a Gaussian process. This indicates that our assumption for $\left\{X_{k}\right\}_{k=1}^{\infty}$ to be subordinated to a Gaussian sequence $\left\{Z_{k}\right\}_{k=1}^{\infty}$ by instantaneous transformations, as required in (1.2), is a substantial restriction in the class of all strictly stationary sequences.

In view of (2.7), Corollary 1 of $\mathrm{Yu}$ [12] proves the statement of the theorem above for a stationary associated sequence $\left\{X_{k}\right\}_{k=1}^{\infty}$ such that $F(\cdot)$ has a bounded density and $\Sigma_{k=1}^{\infty} k^{13 / 2+\delta} \operatorname{Cov}\left(X_{1}, X_{k+1}\right)<\infty$ for some $\delta>0$. (See Yu's paper for the definition of association and for references to previous results for associated sequences.) The two results can be compared when $G(x)=x$ for all $x \in \mathbb{R}$ and $\left\{Z_{k}\right\}_{k=1}^{\infty}$ is a Gaussian sequence as in (1.1) such that $r(k) \geqq 0$ for all $k \in \mathbb{N}$, for in this case the sequence is associated as proved by Pitt [8]. Since $m=1$, our condition is $\sum_{k=1}^{\infty} r(k)<\infty$, while Yu's condition is $\sum_{k=1}^{\infty} k^{13 / 2+\delta} r(k)<\infty$ for some $\delta>0$. (For a Gaussian sequence we do not need $r(\cdot)$ to have the same sign; the result holds whenever $\sum_{k=1}^{\infty}|r(k)|<\infty$. This was also proved earlier by Gastwirth and Rubin [6].)

We do not know whether the theorem remains true for a discontinuous $F(\cdot)$. Our proof breaks down in this case, and it is not even clear that a mean zero Gaussian process $W(\cdot)$ with covariance function as in (2.7) has a version that is almost surely in $\mathscr{D}[-\infty, \infty]$ for a discontinuous marginal distribution function $F(\cdot)$.

\section{Proof}

First we state three lemmas required in proof. Using the Chebyshev inequality, the first one of these is obtained as a special case of Theorem 4.2 in Billingsley [1].

Lemma 1 Let $\left\{W_{n}\right\}_{n=1}^{\infty},\left\{W_{n, \varepsilon}\right\}_{n=1}^{\infty}, V_{\varepsilon}, \varepsilon>0$, and $V$ be real random variables and suppose that (i) $W_{n, \varepsilon} \stackrel{\mathscr{D}}{\rightarrow} V_{\varepsilon}$ for every $\varepsilon>0$, (ii) $\sup \left\{E\left(\left(W_{n}-W_{n, \varepsilon}\right)^{2}\right): n \in\right.$ $\mathbb{N}\} \leqq \varepsilon$, and (iii) $V_{\varepsilon} \stackrel{\mathscr{D}}{\rightarrow} V$ as $\varepsilon \rightarrow 0$. Then $W_{n} \stackrel{\mathscr{D}}{\rightarrow} V$.

Let $\left\{U_{k}\right\}_{k=1}^{\infty}$ be a stationary sequence with marginal distribution that is uniform on the interval $[0,1]$. Introduce the corresponding "uniform" empirical processes

$$
\alpha_{n}^{0}(u):=\frac{1}{\sqrt{n}} \sum_{k=1}^{n}\left[I\left\{U_{k} \leqq u\right\}-u\right], \quad 0 \leqq u \leqq 1, n \in \mathbb{N}
$$

and for any function $f:[0,1] \mapsto \mathbb{R}$, consider its uniform modulus of continuity $w(f, \delta):=\sup \{|f(t)-f(s)|: s, t \in[0,1],|t-s| \leqq \delta\}, 0<\delta<1$.

Lemma 2 If for some positive constant $C>0$, independent of $s, t$ and $n \in \mathbb{N}$,

$$
E\left(\left[\alpha_{n}^{0}(t)-\alpha_{n}^{0}(s)\right]^{4}\right) \leqq C\left[(t-s)^{3 / 2}+\frac{t-s}{n}\right], \quad 0 \leqq s \leqq t \leqq 1,
$$


then for each $\varepsilon>0$ and $\eta>0$ there exist $a \delta \in(0,1)$ and an $n_{0} \in \mathbb{N}$ such that

$$
P\left\{w\left(\alpha_{n}^{0}, \delta\right) \geqq \varepsilon\right\} \leqq \eta \text { for all } n \geqq n_{0} .
$$

Furthermore, (3.3) implies that the sequence $\left\{\alpha_{n}^{0}(\cdot)\right\}_{n=1}^{\infty}$ is tight in $\mathscr{D}[0,1]$, and every subsequential limit of it is almost surely continuous.

Proof. Since $\alpha_{n}^{0}(0)=0$ for every $n \in N$, the second part of the lemma is only a restatement of Theorem 15.5 in Billingsley [1]. The proof of (3.3) parallels the second part of the proof of Theorem 22.1 in Billingsley [1] with a few changes. First, condition (3.2) implies Billingsley's condition (22.15) with the power 2 of $(t-s)$ replaced by $3 / 2$. This implies the validity of $(22.16)$ there, with $m^{2} p^{2}$ replaced by $(m p)^{3 / 2}$ and with $K_{2}=2 K_{4,3 / 2}^{\prime} K_{1}$. For (22.21) to hold true, $\delta>0$ is chosen such that $K_{2} \delta^{1 / 2} / \varepsilon^{5}<\eta$.

Let $\lfloor x\rfloor:=\max \{k \in\{0,1,2, \ldots\}: k \leqq x\}$ be the integer part of $x>0$, so that $\langle x\rangle:=x-|x|$ is its fractional part. The third lemma is a reformulation of Lemma 4.5 of Taqqu [10]. The bound for the constant $K_{p}$ is obtained by first expressing Taqqu's bound for it in the proof of his Lemma 4.5 itself by means of his Corollary 4.2 and then by applying the proof of his Proposition 3.1(ii) to the resulting expression. It is this bound for $K_{p}$ that is important in our applications of the inequality.

Lemma 3 (Taqqu [10]). Let $n, m \geqq 1, p \geqq 2$ and $q \in\{1, \ldots, p\}$ be integers, and suppose that $R:=\max \{|r(k)|: 1 \leqq k \leqq n\}<1 /(p-1)$. If $G_{1}(\cdot), \ldots$, $G_{p}(\cdot)$ are functions in $\mathscr{L}^{2}(\mathbb{R}, \varphi)$ such that at least $q$ among them have Hermite rank greater than or equal to $m$, then

$$
\begin{aligned}
\sum_{1 \leqq n_{1}, \ldots, n_{p} \leqq n}^{\prime}\left|E\left(G_{1}\left(Z_{n_{1}}\right) \ldots G_{p}\left(Z_{n_{p}}\right)\right)\right| \leqq & K_{p} n^{p-\frac{q}{2}}\left[\sum_{k=1}^{n}|r(k)|^{m}\right]^{\frac{q}{2}} \\
& \times\left[\frac{1}{n} \sum_{k=1}^{n}|r(k)|^{m}\right]^{\langle m q / 2\rangle / m},
\end{aligned}
$$

where the summation $\Sigma^{\prime}$ extends over all different indices $1 \leqq n_{1}, \ldots, n_{p} \leqq n$ and $K_{p} \leqq R_{p}\left[E\left(G_{1}^{2}(Z)\right) \ldots E\left(G_{p}^{2}(Z)\right)\right]^{1 / 2}$, where $R_{p}:=2^{p} /\left[1-\{(p-1) R\}^{1 / 2}\right]^{p}$. Proof of the Theorem. Put $\tilde{D}_{u}(\cdot):=\tilde{G}_{u}(\cdot)-u:=I\{F \circ G(\cdot) \leqq u\}-u=$ $I\{F(G(\cdot)) \leqq u\}-u$ and, in analogy to $(1.4)$, write the $\mathscr{L}^{2}(\mathbb{R}, \varphi)$ equations

$$
\tilde{D}_{u}(\cdot)=\tilde{G}_{u}(\cdot)-u=\sum_{k=m\left(\tilde{D}_{u}\right)}^{\infty} \frac{L_{k}(u)}{k !} H_{k}(\cdot), \quad 0 \leqq u \leqq 1,
$$

where $m\left(\tilde{D}_{u}\right) \in \mathbb{N}$ is the Hermite rank of $\tilde{D}_{u}(\cdot)$ and $L_{k}(u)=E\left(H_{k}(Z) \tilde{D}_{u}(Z)\right)$ $=E\left(H_{k}(Z) G_{u}(Z)\right)$. Define $x^{+}=\sup \{y: F(y)=F(x)\}, x \in \mathbb{R}$, and note that $G_{x}(Z)=I\{G(Z) \leqq x\}=G_{x^{+}}(Z)$ almost surely. The continuity of $F$ and the 
definition of $x^{+}$imply that $G_{x+}(\cdot)=I\left\{G(\cdot) \leqq x^{+}\right\}=I\{F(G(\cdot)) \leqq$ $\left.F\left(x^{+}\right)\right\}=I\{F(G(\cdot)) \leqq F(x)\}=\tilde{G}_{F(x)}(\cdot)$ for all $x \in \mathbb{R}$. Thus $G_{x}(Z)=$ $\tilde{G}_{F(x)}(Z)$ almost surely for each $x \in \mathbb{R}$. From the definition of the coefficients $L_{k}(\cdot)$ we see, therefore, that $L_{k}(F(x))=J_{k}(x)$ for all $x \in \mathbb{R}$ and $k \in\{0,1,2, \ldots\}$. Moreover, $\min \left\{m\left(\tilde{D}_{u}(\cdot)\right): 0<u<1\right\}=m$.

Setting $X:=G(Z)$, by the continuity of $F$ the variable $U:=F \circ G(Z)=$ $F(G(Z))=F(X)$ is uniformly distributed on $[0,1]$. Using the terminology at (1.5), this means that the Hermite ranks of the families $\left\{\tilde{D}_{u}(\cdot) 0 \leqq u \leqq 1\right\}$ and $\left\{D_{x}(\cdot): x \in \mathbb{R}\right\}$ are the same. Also, for the uniform empirical processes $\alpha_{n}^{0}(\cdot)$ of (3.1) based on the sequence $\left\{U_{k}=F\left(X_{k}\right)=F\left(G\left(Z_{k}\right)\right)\right\}_{k=1}^{\infty}$ we have the equality $\alpha_{n}(\cdot)=\alpha_{n}^{0}(F(\cdot))$ almost surely, where $\alpha_{n}(\cdot):=$ $\sqrt{n}\left[F_{n}(\cdot)-F(\cdot)\right]$ is the general empirical process in the statement of the theorem. Suppose now that

$$
\alpha_{n}^{0}(\cdot) \stackrel{\mathscr{D}}{\rightarrow} W^{0}(\cdot) \quad \text { in } \quad \mathscr{D}[0,1] \quad \text { and } P\left\{W^{0} \in \mathscr{C}[0,1]\right\}=1,
$$

where $W^{0}(\cdot)$, defined on $[0,1]$, is that case of the limiting process $W(\cdot)$ when the transforming function $G(\cdot)$ is replaced by $F \circ G(\cdot)=F(G(\cdot))$. In other words, $W^{0}(\cdot)$ is a mean-zero Gaussian bridge on $[0,1]$, satisfying $W^{0}(0)=0=W^{0}(1)$ almost surely, with

$$
E\left(W^{0}(u) W^{0}(v)\right)=\sum_{q=m}^{\infty} \frac{L_{q}(u) L_{q}(v)}{q !}\left\{r(0)+2 \sum_{k=1}^{\infty} r^{q}(k)\right\}
$$

for all $0 \leqq u, v \leqq 1$. Let $h: \mathscr{D}[0,1] \mapsto \mathscr{D}[-\infty, \infty]$ be defined by $h \circ f(x):=$ $f(F(x)), x \in[-\infty, \infty] ; f \in \mathscr{D}[0,1]$. Note that $h(\cdot)$ is continuous on $\mathscr{C}[0,1]$. Thus if (3.5) holds, the continuous mapping theorem implies that $\alpha_{n}^{0}(F(\cdot)) \stackrel{\mathscr{Q}}{\rightarrow}$ $W^{0}(F(\cdot))$ in $\mathscr{D}[-\infty, \infty]$. Furthermore, since $L_{k}(F(x))=J_{k}(x)$ for $x \in \mathbb{R}$ and $k \in\{0,1,2, \ldots\}$, we see that the mean-zero Gaussian processes $\left\{W^{0}(F(x))\right.$ : $x \in \mathbb{R}\}$ and $\{W(x): x \in \mathbb{R}\}$ have the same covariance function, and hence the same distribution. Thus, it suffices to prove (3.5) in the set-up (3.4) and (3.6), using condition (2.1).

Fix $u \in[0,1]$, and let $\varepsilon>0$ be arbitrary. By the obvious analogue of (2.6) for $\tilde{D}_{u}(\cdot)$, resulting through $(3.4)$, we see that $u(1-u)=E\left(\tilde{D}_{u}^{2}(Z)\right)=$ $\Sigma_{q=m}^{\infty} L_{q}^{2}(u) / q$ !. Hence by condition (2.1) there exists an $M_{\varepsilon} \in \mathbb{N}$ such that

$$
\sum_{q=M_{\varepsilon}+1}^{\infty} \frac{L_{q}^{2}(u)}{q !} \leqq \frac{\varepsilon}{2 \sum_{k=0}^{\infty}|r(k)|^{m}} \quad \text { and } \quad \lim _{\varepsilon \downarrow 0} M_{\varepsilon}=\infty .
$$

For all small enough $\varepsilon>0$ to make $M_{\varepsilon}>m$, set

$$
W_{n}:=\alpha_{n}^{0}(u)=\frac{1}{\sqrt{n}} \sum_{k=1}^{n} \sum_{q=m}^{\infty} \frac{L_{q}(u)}{q !} H_{q}\left(Z_{k}\right)
$$

and

$$
W_{n, \varepsilon}(u):=\frac{1}{\sqrt{n}} \sum_{k=1}^{n} \sum_{q=m}^{M_{\varepsilon}} \frac{L_{q}(u)}{q !} H_{q}\left(Z_{k}\right)
$$


Using (2.4) and the fact that $|r(k)| \leqq 1$ for all $k \in \mathbb{N}$, for $W_{n, \varepsilon}:=W_{n, \varepsilon}(u)$ we have

$$
\begin{aligned}
E\left(\left(W_{n}-W_{n, \varepsilon}\right)^{2}\right) & =E\left(\left[\frac{1}{\sqrt{n}} \sum_{k=1}^{n} \sum_{q=M_{\varepsilon}+1}^{\infty} \frac{L_{q}(u)}{q !} H_{q}\left(Z_{k}\right)\right]^{2}\right) \\
& =\sum_{q=M_{\varepsilon}+1}^{\infty} \frac{L_{q}^{2}(u)}{q !}\left\{r(0)+2 \sum_{k=1}^{n-1} \frac{n-k}{n} r^{q}(k)\right\} \\
& \leqq 2 \sum_{q=M_{\varepsilon}+1}^{\infty} \frac{L_{q}^{2}(u)}{q !} \sum_{k=0}^{\infty}|r(k)|^{m} \leqq \varepsilon
\end{aligned}
$$

for all $n \in \mathbb{N}$. This means that condition (ii) of Lemma 1 is satisfied. Let $V_{\varepsilon}$ be a mean-zero normal random variable with variance $\sigma_{\varepsilon}^{2}:=\Sigma_{q=m}^{M_{\varepsilon}} L_{q}^{2}(u)\{r(0)+$ $\left.2 \Sigma_{k=1}^{\infty} r^{q}(k)\right\} / q$ !. Since $\sigma_{\varepsilon}^{2} \rightarrow \Sigma_{q=m}^{\infty} L_{q}^{2}(u)\left\{r(0)+2 \sum_{k=1}^{\infty} r^{q}(k)\right\} / q$ ! as $\varepsilon \downarrow 0$ by the very last inequality, we have $V_{\varepsilon} \stackrel{\mathscr{D}}{\rightarrow} W^{0}(u)=: V$ as $\varepsilon \downarrow 0$, that is, condition (iii) of Lemma 1 is also satisfied. Furthermore, since by condition (2.1) and Kronecker's lemma, $\Sigma_{k=1}^{n} k|r(k)|^{q} / n \rightarrow 0$ for every $q \geqq m$, we also have

$$
\begin{aligned}
& \operatorname{Var}\left(W_{n, \varepsilon}\right) \\
& \quad=E\left(W_{n, \varepsilon}^{2}\right)=\sum_{q=m}^{M_{\varepsilon}} \frac{L_{q}^{2}(u)}{q !}\left\{r(0)+2 \sum_{k=1}^{n-1} \frac{n-k}{n} r^{q}(k)\right\} \rightarrow \sigma_{\varepsilon}^{2} \quad \text { for all } \varepsilon>0 .
\end{aligned}
$$

Hence, in view of Lemma 1 , the desired convergence $\alpha_{n}^{0}(u) \stackrel{\mathscr{Q}}{\rightarrow} W^{0}(u)$, for any fixed $u \in[0,1]$, will follow if we show that $W_{n, \varepsilon}(u)$ converges in distribution to some normal random variable for each $\varepsilon>0$ as above.

By the same token, since by (2.1), for each $\varepsilon>0$ as above, we have

$$
\begin{aligned}
E\left(W_{n, \varepsilon}(u) W_{n, \varepsilon}(v)\right) & =\sum_{q=m}^{M_{\varepsilon}} \frac{L_{q}(u) L_{q}(v)}{q !}\left\{r(0)+2 \sum_{k=1}^{n-1} \frac{n-k}{n} r^{q}(k)\right\} \\
& \rightarrow \sum_{q=m}^{M_{\varepsilon}} \frac{L_{q}(u) L_{q}(v)}{q !}\left\{r(0)+2 \sum_{k=1}^{\infty} r^{q}(k)\right\}, \quad 0 \leqq u, v \leqq 1,
\end{aligned}
$$

the desired convergence $\left(\alpha_{n}^{0}\left(u_{1}\right), \ldots, \alpha_{n}^{0}\left(u_{d}\right)\right) \stackrel{\mathscr{D}}{\rightarrow}\left(W^{0}\left(u_{1}\right), \ldots, W^{0}\left(u_{d}\right)\right)$ will follow for any given $\left(u_{1}, \ldots, u_{d}\right) \in[0,1]^{d}, d \in \mathbb{N}$, if we show that $\left(W_{n, \varepsilon}\left(u_{1}\right), \ldots\right.$, $\left.W_{n, \varepsilon}\left(u_{d}\right)\right)$ has a $d$-variate normal limiting distribution. This, in turn, will follow by the Cramér-Wold device [1, p. 49] if the random variable

$$
\sum_{j=1}^{d} b_{j} W_{n, \varepsilon}\left(u_{j}\right)=\frac{1}{\sqrt{n}} \sum_{j=1}^{d} b_{j} \sum_{k=1}^{n} \sum_{q=m}^{M_{\varepsilon}} \frac{L_{q}\left(u_{j}\right)}{q !} H_{q}\left(Z_{k}\right)=\sum_{q=m}^{M_{\varepsilon}} c_{q} S_{n}^{(q)}
$$

has a limiting normal distribution for every fixed vector $\left(b_{1}, \ldots, b_{d}\right) \in \mathbb{R}^{d}$, where $c_{q}:=\sum_{j=1}^{d} b_{j} L_{q}\left(u_{j}\right) / q$ ! and $S_{n}^{(q)}:=\sum_{k=1}^{n} H_{q}\left(Z_{k}\right) / \sqrt{n}$. But this follows from Theorem 1 of Breuer and Major [2] or from Theorem 5 of Giraitis and Surgailis [7]. Hence the finite-dimensional distributions of $\left\{\alpha_{n}^{0}(\cdot)\right\}$ converge to those of the process $W^{0}(\cdot)$. 
The proof of (3.5) will now be complete by an application of the second statement of Lemma 2 and a standard weak-convergence argument, if we show that (3.3) holds for $\alpha_{n}^{0}(\cdot)$. Keeping the first statement of Lemma 2 in mind, we first show that (3.2) holds under the additional condition that $|r(k)| \leqq R$ for all $k \in \mathbb{N}$ for some $0<R<1 / 3$.

The inequality in (3.2) is true when $s=t$ and when $s=0$ and $t=1$. Fix any points $0 \leqq s<t \leqq 1,(s, t) \neq(0,1)$, and define $T(x):=T_{s, t}(x):=\tilde{D}_{t}(x)-$ $\tilde{D}_{s}(x)=\tilde{G}_{t}(x)-\tilde{G}_{s}(x)-(t-s), x \in \mathbb{R}$, so that condition (3.2) is equivalent to

$$
E\left(\left[\sum_{k=1}^{n} T_{s, t}\left(Z_{k}\right)\right]^{4}\right) \leqq C\left[n^{2}(t-s)^{3 / 2}+n(t-s)\right] .
$$

On the other hand, $\left[\sum_{k=1}^{n} T\left(Z_{k}\right)\right]^{4}=\sum_{i=1}^{4} S_{i n}$, where, writing $T^{k}(\cdot):=$ $(T(\cdot))^{k}, k \in \mathbb{N}$,

$$
\begin{aligned}
& S_{1 n}:=\sum_{j=1}^{n} T^{4}\left(Z_{j}\right), \\
& S_{2 n}:=3 \sum_{1 \leqq n_{1}, n_{2} \leqq n}^{\prime} T^{2}\left(Z_{n_{1}}\right) T^{2}\left(Z_{n_{2}}\right)+4 \sum_{1 \leqq n_{1}, n_{2} \leqq n}^{\prime} T^{3}\left(Z_{n_{1}}\right) T\left(Z_{n_{2}}\right), \\
& S_{3 n}:=6 \sum_{1 \leqq n_{1}, n_{2}, n_{3} \leqq n}^{\prime} T^{2}\left(Z_{n_{1}}\right) T\left(Z_{n_{2}}\right) T\left(Z_{n_{3}}\right), \\
& S_{4 n}:=\sum_{1 \leqq n_{1}, n_{2}, n_{3}, n_{4} \leqq n}^{\prime} T\left(Z_{n_{1}}\right) T\left(Z_{n_{2}}\right) T\left(Z_{n_{3}}\right) T\left(Z_{n_{4}}\right)
\end{aligned}
$$

and where the summation $\Sigma^{\prime}$ extends over all different indices that are indicated.

Setting $K:=\sum_{k=1}^{\infty}|r(k)|^{m}$, finite by (2.1), noting that $0<R_{2}, R_{3}, R_{4}<\infty$ for the constants in Lemma 3 by the present side condition that $0<R<1 / 3$, and observing that the rank $m(T)=m\left(T_{s, t}\right) \geqq m$ by the conditions on $(s, t)$ and finally that $E\left(T^{2}(Z)\right)=E\left(T_{s, t}^{2}(Z)\right)=E\left([I\{U \leqq t\}-I\{U \leqq s\}-(t-s)]^{2}\right)=$ $(t-s)\{1-(t-s)\} \leqq t-s$ and $-(t-s) \leqq T(x)=T_{s, t}(x) \leqq 1-(t-s)$ for all $x \in \mathbb{R}$, so that $|T(Z)| \leqq 1$ and $E\left(T^{4}(Z)\right) \leqq E\left(T^{2}(Z)\right) \leqq t-s$, Lemma 3 gives

$$
\begin{aligned}
\left|E\left(S_{4 n}\right)\right| & \leqq K^{4 / 2} K_{4} n^{4-4 / 2} \leqq K^{2} R_{4} n^{2}\left[E\left(T^{2}(Z)\right)\right]^{2} \leqq K^{2} R_{4} n^{2}(t-s)^{2} \\
& \leqq K^{2} R_{4} n^{2}(t-s)^{3 / 2}
\end{aligned}
$$

and

$$
\begin{aligned}
\left|E\left(S_{3 n}\right)\right| & \leqq 6 K^{2 / 2} K_{3} n^{3-2 / 2} \leqq 6 K R_{3} n^{2}\left[E\left(T^{4}(Z)\right)\right]^{1 / 2} E\left(T^{2}(Z)\right) \\
& \leqq 6 K R_{3} n^{2}(t-s)^{3 / 2}
\end{aligned}
$$

Since, noticing that $E\left(I\left\{s<U_{j}, U_{k} \leqq t\right\}\right)=E\left(\left[\tilde{G}_{t}\left(Z_{j}\right)-\tilde{G}_{s}\left(Z_{j}\right)\right]\left[\tilde{G}_{t}\left(Z_{k}\right)-\right.\right.$ $\left.\left.\tilde{G}_{s}\left(Z_{k}\right)\right]\right)=E\left(T\left(Z_{j}\right) T\left(Z_{k}\right)\right)+(t-s)^{2}$, if $j \neq k$ we have

$$
\begin{aligned}
E\left(T^{2}\left(Z_{j}\right) T^{2}\left(Z_{k}\right)\right) & \\
= & E\left(\left[I\left\{s<U_{j} \leqq t\right\}-(t-s)\right]^{2}\left[I\left\{s<U_{k} \leqq t\right\}-(t-s)\right]^{2}\right) \\
= & E\left(I\left\{s<U_{j}, U_{k} \leqq t\right\}\right)[1-2(t-s)]^{2} \\
& +2(t-s)^{3}[1-2(t-s)]+(t-s)^{4} \\
& \leqq \\
& E\left(I\left\{s<U_{j}, U_{k} \leqq t\right\}\right)+3(t-s)^{3} \leqq E\left(T\left(Z_{j}\right) T\left(Z_{k}\right)\right)+4(t-s)^{3 / 2}
\end{aligned}
$$


and

$$
\begin{aligned}
&\left|E\left(T^{3}\left(Z_{j}\right) T\left(Z_{k}\right)\right)\right| \\
&=\left|E\left(\left[I\left\{s<U_{j} \leqq t\right\}-(t-s)\right]^{3}\left[I\left\{s<U_{k} \leqq t\right\}-(t-s)\right]\right)\right| \\
&=\mid E\left(I\left\{s<U_{j}, U_{k} \leqq t\right\}\right)\left[1-3(t-s)+3(t-s)^{2}\right] \\
&-(t-s)^{2}\left[1-3(t-s)+3(t-s)^{2}\right] \mid \\
& \leqq E\left(I\left\{s<U_{j}, U_{k} \leqq t\right\}\right)+(t-s)^{2} \leqq E\left(T\left(Z_{j}\right) T\left(Z_{k}\right)\right)+2(t-s)^{3 / 2},
\end{aligned}
$$

Lemma 3 also gives

$$
\begin{aligned}
\left|E\left(S_{2 n}\right)\right| & \leqq 7 \sum_{1 \leqq n_{1}, n_{2} \leqq n}^{\prime}\left|E\left(T\left(Z_{n_{1}}\right) T\left(Z_{n_{2}}\right)\right)\right|+20 n^{2}(t-s)^{3 / 2} \\
& \leqq 7 K^{2 / 2} K_{2} n^{2-2 / 2}+20 n^{2}(t-s)^{3 / 2} \\
& \leqq 7 K R_{2} n E\left(T^{2}(Z)\right)+20 n^{2}(t-s)^{3 / 2} \\
& \leqq 7 K R_{2} n(t-s)+20 n^{2}(t-s)^{3 / 2}
\end{aligned}
$$

Finally, $\left|E\left(S_{1 n}\right)\right|=n E\left(T^{4}(Z)\right) \leqq n(t-s)$. Collecting the bounds, we obtain (3.7). Hence, by the first statement of Lemma 2, we have (3.3) under the additional condition that $|r(k)| \leqq R$ for all $k \in \mathbb{N}$ for some $0<R<1 / 3$.

Consider now the general case, and fix an $R \in(0,1 / 3)$. Condition (2.1) implies that $r(n) \rightarrow 0$, so we can choose an $n_{0} \in \mathbb{N}$ such that $|r(n)| \leqq R$ if $n \geqq n_{0}$.

Suppose first that $n_{0}=\ell n_{0}$ for some $\ell \in \mathbb{N}$. We split $U_{1}, \ldots, U_{n}$ into $n_{0}$ blocks in the following way: the first block is $U_{1}, U_{n_{0}+1}, U_{2 n_{0}+1}, \ldots, U_{(\ell-1) n_{0}+1}$, the second block is $U_{2}, U_{n_{0}+2}, U_{2 n_{0}+2}, \ldots, U_{(\ell-1) n_{0}+2}, \ldots$, the last block is $U_{n_{0}}$, $U_{2 n_{0}}, \ldots, U_{\ell n_{0}}$. Let $\alpha_{\ell}^{(j)}(\cdot)$ denote the uniform empirical process based on the $\ell$ variables in the $j$ th block, $j=1, \ldots, n_{0}$. Since the indices of each two elements in any block differ by at least $n_{0}$, the absolute value of their covariance is not greater than $R$. Hence the first part of the present tightness proof applies to all of $\alpha_{\ell}^{(1)}(\cdot), \ldots, \alpha_{\ell}^{\left(n_{0}\right)}(\cdot)$ : for each $\varepsilon>0$ and $\eta>0$ there exist a $\delta=\delta(\varepsilon, \eta) \in$ $(0,1)$ and an $\ell_{0}=\ell_{0}(\varepsilon, \eta) \in \mathbb{N}$ such that $P\left\{w\left(\alpha_{\ell}^{(j)}, \delta\right) \geqq \varepsilon\right\} \leqq \eta$ for all $\ell \geqq \ell_{0}$ and all $j=1, \ldots, n_{0}$. Since $\sqrt{n} \alpha_{n}^{0}(\cdot)=\sqrt{\ell} \Sigma_{j=1}^{n_{0}} \alpha_{\ell}^{(j)}(\cdot)$, this implies

$P\left\{w\left(\alpha_{\ell n_{0}}^{0}, \delta\right) \geqq \sqrt{n_{0}} \varepsilon\right\}=P\left\{w\left(\alpha_{n}^{0}, \delta\right) \geqq n_{0}\left(\frac{\ell}{n}\right)^{1 / 2} \varepsilon\right\} \leqq n_{0} \eta \quad$ for all $\ell \geqq \ell_{0}$.

For a completely general $n=\ell n_{0}+i$ with $0 \leqq i \leqq n_{0}-1$ and $\ell \geqq \ell_{0}$, we write

$$
\alpha_{n}^{0}(u)=\frac{1}{\sqrt{n}} \sum_{k=1}^{\ell n_{0}}\left[I\left\{U_{k} \leqq u\right\}-u\right]+\frac{1}{\sqrt{n}} \sum_{k=\ell n_{0}+1}^{\ell n_{0}+i}\left[I\left\{U_{k} \leqq u\right\}-u\right], \quad 0 \leqq u \leqq 1 .
$$

Since, for all $u \in[0,1]$, the absolute value of the second term is not greater than $\sqrt{n_{0} / \ell_{0}}$, which in turn is not greater than $\sqrt{n_{0}} \varepsilon$ if we choose $\ell_{0} \geqq 1 / \varepsilon^{2}$, which is permitted, we see that

$$
P\left\{w\left(\alpha_{n}^{0}, \delta\right) \geqq 2 \sqrt{n_{0}} \varepsilon\right\} \leqq n_{0} \eta \quad \text { for all } n \geqq \ell_{0} n_{0} .
$$


Hence condition (3.3) of Lemma 2 holds in complete generality, and this fact fully establishes (3.5) and hence the theorem.

\section{References}

1. Billingsley, P.: Convergence of probability measures. New York: Wiley 1968

2. Breuer, P., Major, P.: Central limit theorems for non-linear functionals of Gaussian fields. J. Multivariate Anal. 13, 425-441 (1983)

3. Dehling, H., Taqqu, M.S.: The empirical process of some long-range dependent sequences with an application to $U$-statistics. Ann. Statist. 17, 1767-1783 (1989)

4. Deo, C.M.: A note on empirical processes of strong-mixing sequences. Ann. Probab. 1, 870-875 (1973)

5. Dobrushin, R.L., Major, P.: Non-central limit theorems for non-linear functionals of Gaussian fields. Z. Wahrscheinlichkeitstheorie verw. Gebiete 50, 27-52 (1979)

6. Gastwirth, J.L., Rubin, H.: The asymptotic distribution theory of the empiric cdf for mixing stochastic processes. Ann. Statist. 3, 809-824 (1975)

7. Giraitis, L., Surgailis, D.: CLT and other limit theorems for functionals of Gaussian processes. Z. Wahrscheinlichkeitstheorie verw. Gebiete 70, 191-212 (1985)

8. Pitt, L.D.: Positively correlated normal variables are associated. Ann. Probab. 10, 496499 (1982)

9. Sen, P.K.: A note on weak convergence of empirical processes for sequences of $\phi$-mixing random variables. Ann. Math. Statist. 42, 2131-2133 (1971)

10. Taqqu, M.S.: Law of the iterated logarithm for sums of non-linear functions of Gaussian variables that exhibit a long range dependence. $Z$. Wahrscheinlichkeitstheorie verw. Gebiete 40, 203-238 (1977)

11. Taqqu, M.S.: Convergence of integrated processes of arbitrary Hermite rank. Z. Wahrscheinlichkeitstheorie verw. Gebiete. 50, 53-83 (1979)

12. $\mathrm{Yu}, \mathrm{H}$.: A Glivenko-Cantelli lemma and weak convergence for empirical processes of associated sequences. Probab. Theory Related Fields 95, 357-370 (1993) 